\title{
Gut Microbes as a Novel Prognostic and Therapeutic Model in Cancer
}

\author{
Nahla AM Hamed* \\ Anderson Cancer Center, Spain Faculty of Medicine, Alexandria University, Egypt
}

Submission: November 25, 2016; Published: December 07, 2016

*Corresponding author: Nahla AM Hamed, Professor of Hematology, Faculty of Medicine, Alexandria University, Egypt,

Email: drhamedn@hotmail.com

\begin{abstract}
In addition to its association with an expanding list of chronic disorders, commensal microbiota is major player affecting the outcome of carcinogenesis, tumor progression, cancer comorbidities, and response to therapy. Previous studies showed that microbial disturbance by antibiotic exposure severely compromised the efficacy of cyclophosphamide and platinum-based chemotherapeutics. Many of the critical clinical outcomes after allo-HSCT, including overall survival, infections and GVHD are closely linked to changes in the intestinal flora.

Abbreviations: MAMPs: Microbe-Associated Molecular Patterns; LPS: Lipopolysaccharide; GI tract: Gastrointestinal Tract; TLRs: Toll-like Receptors; NLRs: NOD-like Receptors; PRRs: Pattern-Recognition Recepᄀtors; NF-«B: Nuclear Factor Kappa B; TNF: Tumour Necrosis Factor; IL: Interleukin; CDI: Clostridium Difficile; FMT: Fecal Microbiota Transplantation; GVHD: Graft Versus Host Disease; HSCT: Hematopoietic Stem Cell Transplantation; CTX: Cyclophosphamide
\end{abstract}

\section{Introduction}

"Microbiota" is a term applied to describe microorganisms that normally inhabit the human gut, skin, vagina, throat and the upper respiratory tract. Their vast collection of genes is named "microbiome". About 100 trillion microbes inhabit the human gut, which represent 10 folds the number of eukaryotic cells in the body [1].

\section{Development of gut microbiota}

The primary inoculums for vaginally delivered babies is from the mother's vaginal and fecal microbiome while for cesarean section births are from the skin of individuals handling the newborns. The microbiota acquired at birth is of low diversity and unstable. The developing gut microbiota increases in diversity and stability over time [2]. Microbiota assembly towards an adult configuration occurs during the first three years of life. The diversity of adult gut microbiota is higher in vaginally compared to caesarean section-delivered infants. Its composition is individual-specific. In elderly people, gut microbiota is of reduced diversity and increased interpersonal variability [2]. The organism al and gene content of gut communities are shared among family members and transmitted across generations of a kinship [3].

\section{Composition of gut microbiota}

In GI tract, "Friendly" bugs, such as Gram-positive Lactobacilli and Bifid bacteria (constitute more than $85 \%$ of total bacteria) coexist in a complex symbiosis with potential pathogenic bacteria [4]. Their number and complexity gradually increase from the stomach to the colon where $70 \%$ of the total microbes (up to 1011 cells per gram of intestinal content) are present in the colon [1]. Some studies suggest presence of microbiota compartmentalization along GI tract [5].

Gut microbiota composition is influenced by genetic and environmental factors starting early in life [6]. They can also be impacted by host genes, immune system, geography, age, weight, season, diet, illness, stress, lifestyle [7], chronic medications [1] and surgery [7]. Although, gut microbiota display significant inter- and intra-individual variation but on average, they could have long-term stability [7].

\section{Microbiota and immune systems co-evolve}

The microbiota is fueled by dietary macronutrients to produce bioactive compounds. MAMPs such as LPS, peptidoglycan, flagellin or other structural components are sensors of nutrient 
and metabolite availability and convey information about microbes to the host. TLRs, NLRs, inflammasomes, C-type lectins, and RNA-sensing RIG-like helicases are families of innate receptors (PRRs) present on epithelial and immune cells and involved in the recognition of MAMPs [6].

Ligand engagement of these receptors on the apical surface (lumen exposed) epithelium promotes tolerance and healthy inflammatory tone while their activation on the basolateral surface of colonocytes leads to strong pro-inflammatory responses [2].

\section{Functions of microbiota}

Microbiota-derived metabolites signal to distant organs in the body, which enables the gut bacteria to connect to the immune and hormone system, to the brain, to host metabolism and to other functions of the host. This microbe-host communication is essential to maintain vital functions of the healthy host [6].

Data suggest that microbial signals modulate crucial functions of the healthy human body, ranging from host metabolism to brain function. Many of the physiological effects of gut microbiota are caused by their metabolites [6].

The intestinal microbiota can synthesize several vitamins and produce a range of corrinoids [3]. It also affects the absorption of key minerals [3]. Gut microbiota provides nutrients and energy for the host through fermentation of non-digestible dietary components in the large intestine [1]. The microbiota affects expression of host genes involved in nutrient transport and metabolism [3].

The microbiota drives the maturation of the immune system in infancy and contributes to maintenance of its homeostasis during life [1]. Commensal microbiota can profoundly influence the development of the gut mucosal immune system and is able to establish a "cross-talk" with cellular and soluble elements of mucosal immunity [4].

Gut microbiota may contribute to generation of memory alloreactive $\mathrm{T}$ cells and immunoglobulin A- secreting B cells [7]. It playsan important role in the normal architecture of secondary lymphoid organs, differentiation of induced regulatory T cells. Several gut microbe species have been shown to promote expansion or differentiation of Foxp3-expressing regulatory $\mathrm{T}$ cells [7]. Certain commensal bacteria metabolites was highlighted in dictating the extra thymic differentiation of peripheral regulatory $\mathrm{T}$ cells [5]. The intestinal microbiota can also influence systemic immune response [5].

Gut microbiota may be crucial in preventing exogenous pathogen intrusion by direct interaction with pathogenic bacteria and by stimulation of the immune system [4]. Intestinal bacteria are essential for the postnatal development of the enteric nervous system in the mid-distal small intestine [5]. Microbiota also regulates energy expenditure, satiety, glucose homeostasis [1] and metabolic processes. It has been regarded as peacekeepers as well as a neglected endocrine organ [7].

\section{Gut microbiota analyses}

Gut microbiota analyses are often based on fecal mate $\neg$ rial. Yet microbiota composition varies along the intestinal tract and differs even between the intestinal lumen and mucosa-adherent bacteria [6].

Culturing techniques can only detect 10-30\% of gut microbiota [7]. Advanced molecular technologies, such as polymerase chain reaction-denaturing gradient gel electrophoresis and next-generation sequencing technologies, including 16S rRNA sequencing and Meta genomic sequencing have facilitated the analysis of a large number of microorganisms in the gut [7].

\section{Association of microbiota with chronic diseases}

Tight junctions among epithelial cells as well as mechanisms mediated by soluble factors (e.g., antibacterial peptides, antibodies) and innate or adaptive immune cells render mucosal barrier relatively impermeable to microbes and their products. However, some bacterial translocation takes place even under normal physiologic conditions [8]. Infections, inflammation and immune deficient states increased mucosal barrier permeability by altering antimicrobial defense mechanisms and epithelial integrity [4]. Low amounts of bacte $\neg$ rial products such as LPS might reach the lymph and circulation through paracellular diffusion, transcellular transport or co-transport with chylomicrons, which might affect disease development [6].

Alterations of the intestinal microbiota can occur by changes in composition (dysbiosis), function, or microbiotahost interactions [1]. Dysbiosis alters the composition of other colonizing microorganisms by changing the predominance of bacterial species and also directly affects immunity [8]. Several gastrointestinal and extra intestinal diseases can occur when the mutually advantageous bidirectional host-microbe equilibrium in the gut is altered [4].

'Dysbiosis' is associated with an expanding list of chronic disorders such as several gastrointestinal diseases (inflammatory bowel disease, colorectal cancer, or irritable bowel syndrome), as well as extra-intestinal pathologies, such as diseases of the liver, or the respiratory tract (e.g. allergy, bronchial asthma, and cystic fibrosis) atopic dermatiᄀtis, systemic lupus erythematosus, type 1 diabetes, multiple sclerosis, obesity, cardiovascular disease or nervous system diseases etc [7]. The only disease in which a clear causal role of a dysbioticmicrobiota has been demonstrated is CDI infections [1].

Some of these diseases are characterized by heterogeneous disease manifestaᄀtions. It is likely that the different disease phenotypes are associาated with different microbial profiles [6]. Alterations in the number and composition of Bifid bacterium genus species (normal inhabitants of a healthy human gut) is one of the most frequent features present in these diseases [1].

Human studies have identified interactions between host genetics and the microbiome in relation to disease phenotypes. 
For example, NOD2 and CARD9 risk alleles associated with inflammatory bowel disease become manifest only when triggered by the gut micro $\neg$ biome indicating that genetic predisposition to diseases can depend on the microbiome [9].

\section{Impact of gut microbiota on cancer prevention}

Ellagic acid is a cancer-preventive antioxidant exhibiting anti estrogenic and anti-COX2 activities when metabolized by colonic microbiota into urolithins. Another polyphenol called 'daidzein' is metabolized by gut microbiota into equal and is detected only in a fraction of individuals harboring sulfate-reducing bacteria. It may protect against breast and prostate cancer, mostly in Asia [5].

\section{Impact of gut microbiota on cancer progression and outcome}

The commensal microbiota is a major player affecting carcinogenesis outcome, tumor progression, cancer comorbidities, and response to therapy by setting the inflammatory/immune tone, modulating host response to oncogenic pathogens, cancer associated inflammation, and tumor-induced tissue damage [8].

Myeloid cells, a major component of the tumor micro environment, respond to environmental factors including signals derived from commensal microbes. Myeloid cells play divergent dual roles in cancer. They can induce antitumor immune responses, but mostly they promote immune evasion, tumor progression, and metastasis formation [10].

A variety of microbial ligands stimulate activation of NF- $\kappa B$ and downstream pro-inflammatory cytokines such as TNF- $\alpha$ and IL-1 [2]. TNF is a potent tumor promoting cytokine that stimulates the metastatic spread of ectopic colorectal tumors [10].

Elevated production of IL-17, in response to translocation commensal bacteria or their disintegration products was linked to rapid progression from a controllable stage of colorectal cancer to metastatic disease in humans. Elevated IL-17 production has also been linked to therapy failure and most recently shown to antagonize anti-angiogenic treatment [10].

\section{Evidence comes from the following observations}

A. Certain commensalisms are involved in the natural immune surveillance of malignancies exposed to the portals of entry (such as ulcerated melanoma). Hence, lung tumors presenting tertiary lymphoid organogenesis exhibit a more favorable prognosis, perhaps owing to chronic stimulation with environmental microorganisms [5].

B. In humans, some epidemiologic studies suggested a dose-dependent association between increased cumulative days of antibiotic use and risk of breast cancer [11].

C. A bacterial driver-passenger model was proposed for microbial involvement in colorectal cancer development.
Distinct indigenous intestinal bacteria, 'driver bacteria' would create DNA damage and drive genome instability to initiate the first steps of tumorigenesis [5]. Bacterial drivers may progressively disappear in favor of opportunistic bacteria 'passenger bacteria', which then overwhelm the intestinal niche alterations and corrupt the local innate immunity. According to this model bacteria must be incorporated into the genetic paradigm of cancer progression [5].

D. It is speculated that the mammalian gut microbiota could also influence cancer susceptibility and recurrence based on a study done in mice [12].

\section{Impact of gut microbiota on cancer chemotherapy}

The intact commensal gut microbiota influences early response to cancer immunotherapy and chemotherapy by affecting differentiation and functions of myeloid-derived cells in the tumor microenvironment. The efficacy of chemotherapeutic agents might also depend on microbiota-mediated innate and adaptive immune responses [13].

Does prophylactic antibiotic treatment taken by cancer patients before receiving chemotherapy interferes with the efficacy of chemotherapeutics?

Many genotoxic cancer drugs lead to mucositis, gut barrier deterioration and bacterial translocation. These drugs cause also neutropenia which leads to bacterial translocation across the gut mucosa and severe systemic infections that will require antibiotics [10]. A previous study shows that subcutaneous tumors fail to respond to immunotherapy and platinum chemotherapy after antibiotic treatment [14]. Another study reports that cyclophosphamide effect on antitumor immune response relies on the presence of a 'healthy' gut microbiota [15]. These studies showed that microbial disturbance by antibiotic exposure severely compromised the efficacy of CTX and platinum-based chemotherapeutics $[14,15]$.

CTX facilitates priming of effector pTh17 and memory Th1 cell responses directed against distinct commensals in distant secondary lymphoid organs thus facilitating the accumulation of Th1 helper T cells in tumor beds as well as tumor regression [15]. Iida et al. [14] observed that the presence of the commensals Alistipesand Ruminococcuscor relate positively with the capacity of tumor-associated myeloid cells to secrete TNF- $\alpha$, thereby enhancing anticancer effect.

These findings were mostly generated using mouse xenograft cancer models and as such may have limited relevance to human cancer. However, Antibiotic use in humans rarely leads to nearly complete depletion of the gut microflora, and any dysbiosis that ensues is usually transient [10].

It is tempting to speculate that the variable response to cancer therapy observed in patients may be in part due to different microbiota composition, affecting the inflammatory tone and myeloid-cell functions in the tumor microenvironment. 
Furthermore, the same bacterial species may have opposite effects in different types or at different stages of therapy. It seems that the cytokines modulated by the gut microbiome can have opposing effects on tumor growth and outcome of cancer therapy [8].

This explanation would offer the possibility to improve the effectiveness of immune therapy, chemotherapy, and radiotherapy by using 'immunostimulatory microorganisms' to alleviate the deleterious effect of microbiota depletion, targeting the pathways by which the microbiota communicates with inflammatory cells, or by targeting the molecular mechanisms that restrict the functions of myeloid cells and antigen presenting cells [8].

\section{Impact of gut microbiota on GVHD}

Most transplant patients are put on special diet during hospitalization which can impact gut microbiota [7]. Studies in mouse and human have indicated that many of the critical clinical outcomes after allo-HSCT, including overall survival, infections and GVHD are closely linked to changes in the intestinal flora [16].

Inferring the status of the whole gut microbiota by rectal samples only may be a challenge due to significant difference in the diversity and population of microbiota along GI tract. Higher phylogenetic diversity was found in gastric, duodenal, large intestinal and fecal samples than jejunum and ileum samples [7]. A greater proportion of anaerobes, such as Bacteroidaceae, Prevotellaceae, Rikenellaceae, Lachnospiraceae, and Ruminococcaceae were found in the large intestine and feces. However, a larger proportion of Lactobacillaceae were found in the stomach and small intestine [7].

In the weeks following allo-HSCT, a decrease in commensally intestinal bacterial diversity occurs in most patients. This is often accompanied by dominance of certain bacteria and significantly decreased overall survival. About one-half of bacteremic episodes after allo-HSCT are preceded by intestinal domination of a corresponding organism. Domination by Enterococcus spp, Streptococci and Proteobacteria account for $30 \%$ or more of fecal $16 \mathrm{~S}$ ribosomal sequences and are important clinically [16].

Enterococci were more pronounced with antibiotic prophylaxis and treatments of neutropenic infections [7]. Enterococci shift was prominent in those who subsequently developed or suffered from active gastrointestinal GVHD [7].

Loss of microbial diversity and Clostridiales and expansion of Lactobacillales in intestinal microbiota was observed in mouse models and patients with GVHD after BMT. Eliminating Lactobacillales from the gut flora in mice before BMT could cause GVHD. When reintroducing a predominant species of Lactobacillus, GVHD was alleviated. There was a close correlation between alterations in the intestinal microbiota and GVHD severity and mortality outcomes. Patients with significantly worse mortality outcomes had lower gut microbial diversity [7].
A key event in the initiation of inflammation during GVHD is the activation of PRRs, on hematopoietic cells [17], mucosal cells, immune and gut endothelial cells by microbes or microbial products produced by the intestinal flora such as LPS. The liver can maintain tolerance against harmless antigens derived from commensally bacteria, even if commensally bacteria escape from the gut [7]. The most studied PRRs are TLRs and NOD-like receptors. Polymorphisms of the genes encoding NOD2andTLR4 are associated with a higher incidence of GVHD in HSC transplant recipients [17].

Intracellular adaptors such as MyD88 are indispensable in transferring PRR signaling information. MyD88 is a downstream molecule of all TLRs except TLR3. PRR signaling in cells can promote expression of major histo compatibility complex and costimulatory molecules, particularly on antigen-presenting cells and some endothelial cells [7]. As a result, cytokines such as TNF, type I interferons, IL- 1 and IL- 6 are produced resulting in local tissue inflammation, migration of leukocytes, presentation of host antigens, and eventually anti host reactivity of donor $\mathrm{T}$ cells [7].

\section{Gut microbiota as a novel therapeutic target}

There are various approaches to shape the gut microbiota to restore host-microbial balance, including personalized probiotic, prebiotics (fiber), vitamin/mineral supplementation, dietary, FMT, and/or the use of antibiotics [18].Promising and encouraging results have been obtained with antibiotics, probiotics and prebiotics. Gut microbiota regulation has the potential to be a novel therapeutic target to prevent or treat complications after allogeneic transplantation [7].

A prebiotic approach involves administration of certain foods or food components rich in poorly digested or poorly absorbed carbohydrates and fibres that are metabolized by beneficial commensal bacteria. Administration of prebiotics may augment the production of metabolic products that result from their fermentation [16].

Probiotics are live microorganisms ingested either through diet, e.g. yogurt or in the form of a probiotic supplement [2]. In a postbiotic intervention, one would administer a bacterial metabolic product that mediates some benefit. Several Clostridial metabolites, including indole and its derivatives can negatively regulate the growth of potential pathogens such as Gram-negative bacteria and Candida [16].

FMT consists of engraftment of microbiota from a healthy donor(s) into a recipient to restore normal gut microbial community structure, with the aim of recovering metabolic and immunologic balance [1]. Fecal transplant may have also anti-inflammatory and pro-inflammatory effects on mucosal inflammation. FMT is used when specific bacterial species or their combination was not the best possibility to prevent or cure disease. Fecal transplants change the composition of the recipient gut microbiota to resemble that of the donor for at least several weeks [8]. 
FMT is a very successful therapy to treat CDI and is a current, recommended treatment for relapsing and non-responding CDI [1]. Fecal transplants have also been proposed as a treatment for inflammatory bowel diseases and metabolic disorders; transfer of intestinal microbiota from lean donors has been shown to increase sensitivity to insulin in patients with metabolic syndrome. In theory, fecal transplants could treat dysbiosis that are associated with cancer comorbidities and could contribute to optimal response to cancer therapy [8].

An alternative to the use of bacterial preparations is to administer bacterial-derived or bacterial-induced products that modulate the immune system. Ligands for TLRs or other innate receptors are being developed for clinical use and could be used in combination with cancer therapies [19]. Strategies aiming at reprogramming the tumor microenvironment represent a promising immunotherapy approach [8].

\section{Conclusion and Future Prospect}

Better management of cancer patients requires diagnosis of relation of dysbiosis to diet, medications and exercise, and so on), selection of the appropriate 'immunogenic probiotics' to over express specific functions, synergize prebiotics with probiotics to set a healthy intestine that has been compromised by DNAdamaging agents, and by monitoring the immune responses raised against the relevant commensals [5].

\section{References}

1. Tojo R, Suarez A, Clemente MG, Reyes-Gavilan CG, Margolles A, et al. (2014) Intestinal microbiota in health and disease: Role of bifidobacteria in gut homeostasis. World J Gastro enterol 20(41): 15163-15176.

2. Peterson CT, Sharma V, Elmen L, Peterson SN (2014) Immune homeostasis, dysbiosis and therapeutic modulation of the gut microbiota. Clinical and Experimental Immunology 179: 363-377.

3. Kau AL, Ahern PP, Griffin NW, Goodman AL, Gordon J, et al. (2011) Human nutrition, the gut microbiome and the immune system. Nature 474: 327-336.

4. Purchiaroni F, Tortora A, Gabrielli M, Bertucci F, Gigante, G et al. (2013) The role of intestinal microbiota and the immune system. European Review for Medical and Pharmacological Sciences 17: 323-333.
5. Viaud S, Daille re R, Boneca IG, Lepage P, Langella P, et al. (2015) Gut microbiome and anticancer immune response: really hot Sh*t!. Cell Death and Differentiation 22: 199-214.

6. Schroeder BO, Backhed F (2016) Signals from the gut microbiota to distant organs in physiology and disease. Nature Medicine 22 (10): 10779-10789.

7. Wang W, Xu S, Ren Z, Jiang J, Shusen Z (2015) Gut microbiota and allogeneic transplantation. J Transl Med 13: 275-285.

8. Goldszmid RS, Dzutsev A, Viaud S, Zitvogel L, Restifo NP et al. (2015) Microbiota modulation of myeloid cells in cancertherapy. Cancer Immunol Res 3(2): 103-109.

9. Bonder MJ, Kurilshikov A, Tigchelaar EF, Mujagic Z, Imhann F et al. (2016) The effect of host genetics on the gut microbiome. Nature Genetics 48: 1407-1412.

10. Karin M, Jobin C, Balkwill F (2014) Chemotherapy, immunity and microbiota- a new triumvirate? Nat Med 20(2): 126-127.

11. Velicer CM, Susan R, Johanna W. L, John DP, Carol AR (2004) Antibiotic use in relation to the risk of breast cancer. J Am Med Assoc 291: 827835.

12. Kottke T, Nicolas B, Rosa Maria D, Oliver D, Diana, et al. (2013) Detecting and targeting tumor relapse by its resistance to innate effectors at early recurrence. Nat Med 19: 1625-1631.

13. Greenhill C (2014) Anti-cancer therapies affected by gut microbiota. Nature Reviews Gastroenterology \& Hepatology 11: 1.

14. Iida N, Dzutsev A, Stewart CA, Smith L, Bouladoux N, et al. (2013) Commensal bacteria control cancer response to therapy by modulating the tumor microenvironment. Science 342: 967-970.

15. Viaud S, Saccheri F, Mignot G, Yamazaki T, Daillere R, et al. (2013) The intestinal microbiota modulates the anticancer immune effects of cyclophosphamide. Science 342(6161): 971-976.

16. Peled JU, Jenq RR, Holler E, Van den Brink MRM (2016) Role of gut flora after bone marrow transplantation. Nature Microbiology 1: 1-2.

17. Penack O, Holler E, Van den Brink MRM (2010) Graft-versus-host disease: regulation by microbe-associated molecules and innate immune receptors. Blood 115(10): 1865-1872.

18. Sun J, Chang EB (2014) Exploring gut microbes in human health and disease: Pushing the envelope. Genes Dis 1(2): 132-139.

19. Ridnour LA, Cheng RY, Switzer CH, Heinecke JL, Ambs S, et al. (2013) Molecular pathways: toll-like receptors in the tumor microenvironment-poor prognosis or new therapeutic opportunity. Clin Cancer Res 19(6): 1340-1346.

\begin{tabular}{|l|}
\hline \multicolumn{1}{|c|}{ Your next submission with Juniper Publishers } \\
will reach you the below assets \\
- Quality Editorial service \\
- Swift Peer Review \\
- Reprints availability \\
- E-prints Service \\
- Manuscript Podcast for convenient understanding \\
- Global attainment for your research \\
- Manuscript accessibility in different formats \\
( Pdf, E-pub, Full Text, Audio) \\
- Unceasing customer service \\
Track the below URL for one-step submission \\
https://juniperpublishers.com/online-submission.php \\
\hline
\end{tabular}

